\title{
TITLE:
}

\section{OCEANOGRAPHIC DATA AT THE S. M. B. L., 1972}

$\operatorname{AUTHOR}(\mathrm{S})$ :

\section{CITATION:}

OCEANOGRAPHIC DATA AT THE S. M. B. L., 1972. PUBLICATIONS OF THE SETO MARINE BIOLOGICAL LABORATORY 1976, 22(6): 409-415

ISSUE DATE:

1976-02-28

URL:

http://hdl.handle.net/2433/175908

RIGHT: 


\section{OGEANOGRAPHIC DATA AT THE S. M. B. L., 1972}

Measurements of the surface sea water temperature were made every day at 9:00 in the morning at the fixed site on the south coast of the laboratory ground near the water intake of the laboratory by
Mr. Yoshirô Kashiyama,
Mr. Sôichi Moriyama,
Mr. Mitsuru Ohta,
Mr. Sadayoshi Ohtsuki,
Mr. Shôzô Sakai,
Mr. Hidetomo Tanase, and
Mr. Yoshikazu Yamamoto.

The salinity was measured by salinometer (Tsurumi Co., Ltd., Type E2).

Meteorological observations were made similarly in the laboratory yard by

Miss Junko Kondô.

The necessary correction after formal manuals of oceangraphical and meteorological observations, as well as the typewriting of tables, were made by

Dr. Shin-ichirô Fuse.

Weather is shown in Beaufort notation, and wind force in Beaufort scale. The codes used are:

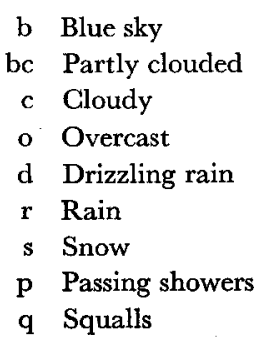

0 Calm, less than $0.3 \mathrm{~m} / \mathrm{sec}$

1 Light air, $0.3 \sim 1.5 \mathrm{~m} / \mathrm{sec}$

2 Light breeze, $1.6 \sim 3.3 \mathrm{~m} / \mathrm{sec}$

3 Gentle breeze, $3.4 \sim 5.4 \mathrm{~m} / \mathrm{sec}$

4 Moderate breeze, $5.5 \sim 7.9 \mathrm{~m} / \mathrm{sec}$

5 Fresh breeze, $8.0 \sim 10.7 \mathrm{~m} / \mathrm{sec}$

Summarizing the data, the following annual values are obtained for 1972:

\begin{tabular}{lcll} 
& \multicolumn{2}{c}{ Lowest } & \multicolumn{2}{c}{ Highest } & Mean \\
Air temperature $\left({ }^{\circ} \mathrm{C}\right)$ & $5.4 \quad$ (Jan. 18) & 31.0 (Sept. 6) & 18.0 \\
Water temperature $\left({ }^{\circ} \mathrm{C}\right)$ & 12.50 (Feb. 8) & 29.20 (Aug. 5) & 18.50 \\
Salinity (\%o) & 27.005 (June 16) & 35.101 (Jan. 14) & 33.642 \\
Total precipitation $(\mathrm{mm})$ & 2702.9 & &
\end{tabular}


JANUARY

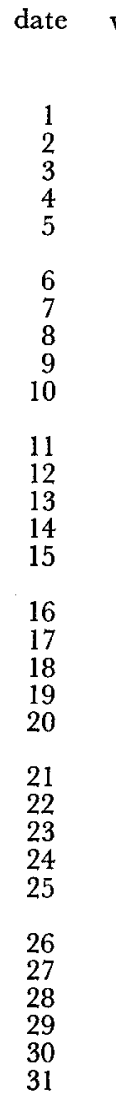

mean precipi- wind perature

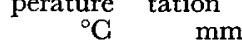

11.3
8.8
6.8
10.2
14.8

8.4

\begin{tabular}{lr}
8.4 & $\mathrm{E}$ \\
6.8 & $\mathrm{NW}$ \\
\hline
\end{tabular}

E 1
NW 2

$2 \overline{2.0}$

$\begin{array}{rr}\mathbf{N} & 1 \\ \mathbf{W} & 1 \\ \mathrm{~W} & 1\end{array}$

9.8
9.4
9.2
8.3
11.7

$\frac{2.5}{0.7}$

NW 2

$\begin{array}{rl}\mathrm{E} & 1 \\ \mathrm{NE} & 1\end{array}$

$\begin{array}{ll}13.3 & 2.7 \\ 13.4 & 2.7\end{array}$

11.1
9.6
9.7

-

$\mathrm{E} 1$
$\mathrm{~N} 2$
$\mathrm{~N} 2$
$\mathrm{~N}$

9.4
8.2
5.4
8.8
13.4

-

NW 1

NW 2

$N$
-0
-0

$-0$

$\begin{array}{rrr}11.7 & - & -0 \\ 10.1 & - & \mathrm{N} 2 \\ 8.9 & - & \end{array}$

8.9
14.3
17.8

18.9

W 1

10.9
9.1
9.0
6.9
10.5
9.2

10.4

0.7

NW 2

NW 1

NW 2
-0 water tem- salinity perature

17.24 16.68 $14.70 \quad 34.732$ $14.75 \quad 34.310$ $15.45 \quad 34.746$ $15.55 \quad 34.604$

17.25 $18.50 \quad 34.756$ $16.62 \quad 34.828$ $17.30 \quad 34.984$

$17.70 \quad 34.532$ $16.80 \quad 34.975$ $16.50 \quad 34.886$ $16.70 \quad 35.101$ $16.70 \quad 34.919$

$\begin{array}{ll}15.52 & 34.773 \\ 15.20 & 34.962\end{array}$

$\begin{array}{ll}15.20 & 34.962 \\ 16.60 & 34.975\end{array}$

$16.60 \quad 34.975$

$\begin{array}{ll}17.10 & 34.964 \\ 17.70 & 34.869\end{array}$

$17.20 \quad 34.927$

$16.30 \quad 35.003$

$17.02 \quad 34.982$

$16.30 \quad 34.886$

$\begin{array}{ll}18.25 & 34.768\end{array}$

16.70

15.95
15.50

\subsection{8}

34.924
34.564

34.564

34.484

34.503

34.426
34.684

16.53

34.778

FEBRUARY

date weather air temair tem- precipi-
perature tation $\begin{array}{ll}{ }_{15.4}^{\circ} \mathrm{C} & \mathrm{mm} \\ 11.9 & \end{array}$

wind

1
2
3
4
5

6

6
7
8

8
9
10

12
13
14
15

16
17
18
19
20

2

21
22
23
24
25

24
25

26
27
28
29

mean

$\mathrm{d}$
$\mathrm{o}$
$\mathrm{o}$
$\mathrm{d}$
$\mathrm{c}$
$\mathrm{o}$
$\mathrm{o}$
$\mathrm{bc}$
$\mathrm{o}$
$\mathrm{d}$
$\mathrm{b}$
$\mathrm{b}$
$\mathrm{r}$
$\mathrm{bc}$
$\mathrm{bc}$
$\mathrm{r}$
$\mathrm{c}$
$\mathrm{c}$
$\mathrm{r}$
$\mathrm{bc}$
$\mathrm{bc}$
$\mathrm{o}$
$\mathrm{o}$
$\mathrm{bc}$
$\mathrm{b}$

$\mathrm{o}$
$\mathrm{o}$
$\mathrm{bc}$
$\mathrm{o}$

9.1 water tem- salinity perature

17.25

$16.72-34.181$

$\begin{array}{rrrrr}11.6 & - & \text { NW } 2 & 15.80 & 34.577 \\ 9.2 & 35.5 & -0 & 15.40 & 34.419 \\ 9.4 & 5.4 & \text { N } 1 & 16.00 & 34.574\end{array}$

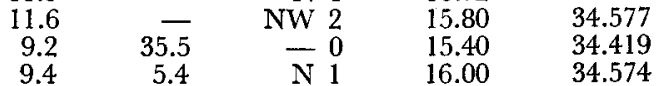

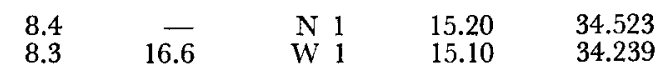

$\begin{array}{lllll}6.7 & - & \mathrm{N} & 15.10 & 34.2313\end{array}$

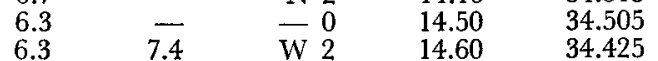

$\begin{array}{lllll}9.9 & 0.3 & \text { NW } 2 & 16.48 & 34.735\end{array}$

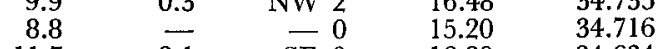

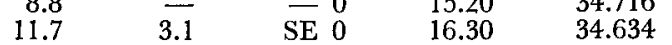

$\begin{array}{llll}8.4 & 30.0 & W & 15.40\end{array}$

$\begin{array}{lrlll}8.4 & 30.0 & \text { W } 2 & 15.40 & 34.542 \\ 9.6 & - & \text { W } 2 & 14.60 & 34.512\end{array}$

$\begin{array}{rrrrr}7.3 & 0.2 & -0 & & \\ 10.9 & 5.2 & \mathrm{~N} 2 & 15.60 & 34.112\end{array}$

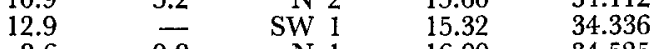

$\begin{array}{lllll}8.6 & 0.8 & \text { N } 1 & 16.00 & 34.525 \\ 7.4 & 0.1 & \text { N } 2 & 14.60 & 34.651\end{array}$

$\begin{array}{lllll}7.0 & - & \text { NW } 2 & 14.80 & 34.838\end{array}$

$9.4 \quad-\quad$ NW $113.00 \quad 34.771$

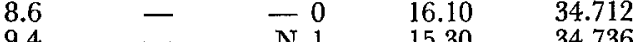

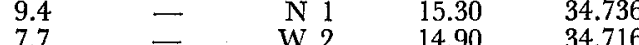

$\begin{array}{lllll}9.4 & 58.0 & \text { E } ~ & 15.50 & 34.629\end{array}$

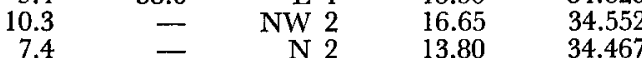

$\begin{array}{lllll}7.4 & - & \mathrm{N} 2 & 13.80 & 34.467 \\ 6.1 & - & -0 & 14.70 & 34.446\end{array}$

15.43

34.543 
MARCH

\begin{tabular}{|c|c|c|c|c|c|c|}
\hline date & weather & $\begin{array}{l}\text { air tem- } \\
\text { perature } \\
{ }^{\circ} \mathbf{C}\end{array}$ & $\begin{array}{l}\text { precipi- } \\
\text { tation } \\
\text { mm }\end{array}$ & wind & $\begin{array}{l}\text { water tem- } \\
\text { perature } \\
{ }^{\circ} \mathrm{C}\end{array}$ & salinity \\
\hline $\begin{array}{l}1 \\
2 \\
3 \\
4 \\
5\end{array}$ & $\begin{array}{l}\mathrm{bc} \\
\mathrm{b} \\
\mathrm{c} \\
\mathrm{b} \\
\mathrm{b}\end{array}$ & $\begin{array}{l}6.6 \\
7.6 \\
6.8 \\
9.2 \\
7.4\end{array}$ & $\begin{array}{l}\bar{z} \\
\bar{E} \\
-\end{array}$ & $\begin{aligned} \text { NW } & 3 \\
\text { NNW } & 3 \\
\text { NNW } & 3 \\
\text { NW } & 3 \\
\text { NW } & 2\end{aligned}$ & $\begin{array}{l}14.47 \\
14.60 \\
14.12 \\
14.42 \\
15.07\end{array}$ & $\begin{array}{l}34.683 \\
34.698 \\
34.719 \\
34.733 \\
34.669\end{array}$ \\
\hline $\begin{array}{r}6 \\
7 \\
8 \\
9 \\
10\end{array}$ & $\begin{array}{l}\mathrm{b} \\
\mathrm{b} \\
\mathrm{b} \\
\mathrm{o} \\
\mathrm{o}\end{array}$ & $\begin{array}{r}9.8 \\
10.6 \\
10.1 \\
10.7 \\
9.4\end{array}$ & $\bar{E}$ & 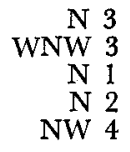 & $\begin{array}{l}14.12 \\
14.73 \\
14.52 \\
14.52 \\
14.32\end{array}$ & $\begin{array}{l}34.665 \\
34.675 \\
34.634 \\
34.178 \\
34.601\end{array}$ \\
\hline $\begin{array}{l}11 \\
12 \\
13 \\
14 \\
15\end{array}$ & $\begin{array}{l}\mathrm{bc} \\
\mathbf{o} \\
\mathrm{c} \\
\mathrm{bc} \\
\mathrm{bc}\end{array}$ & $\begin{array}{r}10.3 \\
10.8 \\
7.4 \\
9.8 \\
9.1\end{array}$ & $\begin{array}{l}- \\
z \\
-\end{array}$ & $\begin{array}{rr}\mathrm{N} & 2 \\
\mathrm{NW} & 1 \\
\mathrm{~N} & 2 \\
\mathrm{NW} & 1 \\
\mathrm{NW} & 2\end{array}$ & $\begin{array}{l}14.35 \\
14.66 \\
14.10 \\
14.10 \\
13.70\end{array}$ & $\begin{array}{l}34.612 \\
34.714 \\
34.565 \\
34.487 \\
34.484\end{array}$ \\
\hline $\begin{array}{l}16 \\
17 \\
18 \\
19 \\
20\end{array}$ & $\begin{array}{l}\mathrm{b} \\
\mathrm{r} \\
\mathrm{b} \\
\mathrm{o} \\
\mathrm{o}\end{array}$ & $\begin{array}{r}9.3 \\
13.4 \\
14.9 \\
15.8 \\
19.0\end{array}$ & $\begin{array}{r}2 \overline{26.7} \\
6.7 \\
\overline{12.6}\end{array}$ & 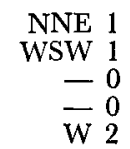 & $\begin{array}{l}14.39 \\
15.02 \\
14.98 \\
15.70 \\
16.05\end{array}$ & $\begin{array}{l}34.401 \\
33.400 \\
34.058 \\
34.040 \\
33.670\end{array}$ \\
\hline $\begin{array}{l}21 \\
22 \\
23 \\
24 \\
25\end{array}$ & $\begin{array}{r}\mathrm{bc} \\
\mathrm{bc} \\
\mathrm{r} \\
\mathrm{r} \\
\mathrm{c}\end{array}$ & $\begin{array}{l}12.3 \\
16.3 \\
16.1 \\
15.4 \\
13.9\end{array}$ & $\begin{array}{r}3.2 \\
17.0 \\
25.0 \\
4.4\end{array}$ & $\begin{array}{rr}\text { NW } & 2 \\
- & 0 \\
\text { ENE } & 1 \\
\text { NW } & 2 \\
-0\end{array}$ & $\begin{array}{l}15.30 \\
14.70 \\
15.39 \\
16.21 \\
16.10\end{array}$ & $\begin{array}{l}33.675 \\
34.450 \\
33.544 \\
33.080 \\
33.729\end{array}$ \\
\hline $\begin{array}{l}26 \\
27 \\
28 \\
29 \\
30 \\
31\end{array}$ & $\begin{array}{r}r \\
b \\
b \\
b c \\
r \\
b c\end{array}$ & $\begin{array}{l}11.3 \\
13.0 \\
13.4 \\
16.9 \\
18.3 \\
16.6\end{array}$ & $\begin{array}{r}22.0 \\
4.4 \\
\overrightarrow{9.8} \\
17.5 \\
-\end{array}$ & $\begin{array}{rr}\text { NW } & 2 \\
\text { NW } & 1 \\
- & 0 \\
\text { NE } & 1 \\
\text { S } & 3 \\
\text { NW } & 3\end{array}$ & $\begin{array}{l}15.94 \\
14.32 \\
15.85 \\
17.03 \\
18.49 \\
18.33\end{array}$ & $\begin{array}{l}33.907 \\
33.563 \\
33.886 \\
34.187 \\
34.138 \\
34.031\end{array}$ \\
\hline mean & & 12.0 & & & 15.15 & 34.222 \\
\hline
\end{tabular}

APRIL

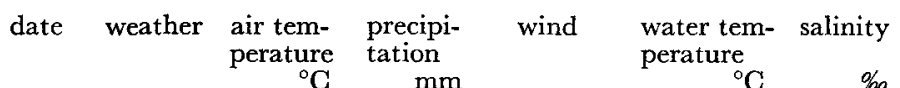

$\begin{array}{rrrrrrr}1 & \text { c } & 6.8 & - & \text { NW } 2 & 15.80 & 34.149 \\ 2 & \text { bc } & 8.7 & 0.4 & \text { N } 1 & 15.10 & 34.10 \\ 3 & \text { o } & 10.8 & -5 & \text { T } & 15.54 & 33.92 \\ 4 & \text { o } & 7.3 & 4.5 & \text { W } & 16.75 & 33.98 \\ 5 & \text { bc } & 15.8 & - & \text { E } 1 & 17.21 & 34.11\end{array}$

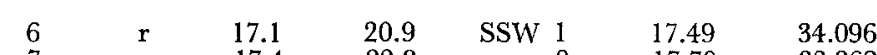
$\begin{array}{lllllll}7 & \mathrm{o} & 17.4 & 22.8 & -0 & 17.70 & 33.862 \\ 8 & \mathrm{r} & 13.6 & 12.0 & -0 & 17.75 & 33.889\end{array}$ $\begin{array}{rrrrrrr}9 & \circ & 10.5 & 17.0 & \text { NW } 2 & 15.95 & 34.244 \\ 10 & \mathrm{~b} & 11.6 & 44.0 & \text { NW } 1 & 15.89 & 34.263\end{array}$

$\begin{array}{lllllll}11 & \circ & 12.6 & - & \text { NE } 2 & 15.00 & 34.166\end{array}$

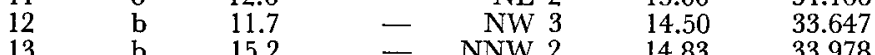

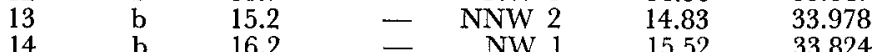

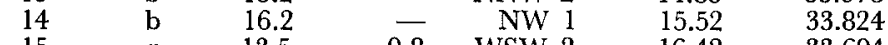
$\begin{array}{lllllll}15 & \mathrm{r} & 13.5 & 0.8 & \text { WSW 2 } & 16.42 & 33.694\end{array}$

$\begin{array}{lllllll}16 & b & 18.1 & 27.6 & -0 & 16.62 & 33.667\end{array}$ $\begin{array}{lllllll}17 & \text { bc } & 19.8 & 0.1 & -0 & 16.72 & 33.657 \\ 18 & \text { bc } & 20.4 & - & -0 & 17.27 & 33.506\end{array}$

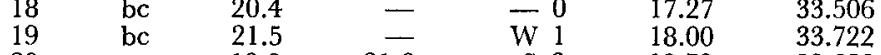
$\begin{array}{lllllll}20 & \mathrm{r} & 19.0 & 21.0 & \mathrm{~S} 2 & 18.72 & 32.658\end{array}$ $\begin{array}{rrrrrrr}21 & o & 18.4 & 2.2 & \text { NNW } 3 & 17.02 & 33.153\end{array}$

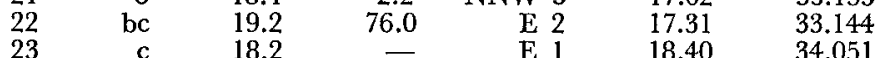

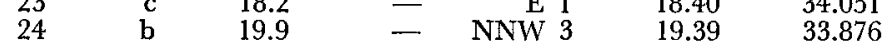
$\begin{array}{lllllll}25 & 0 & 18.3 & - & -0 & 19.12 & 33.640\end{array}$ $\begin{array}{lllllll}26 & 0 & 19.9 & 0.6 & -0 & 20.01 & 33.664\end{array}$

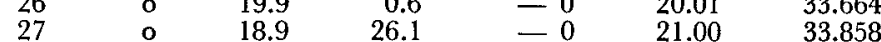

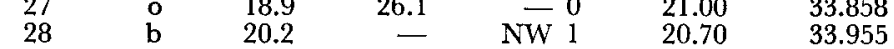

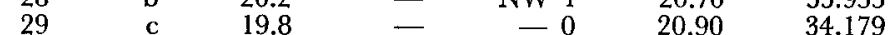

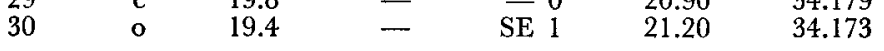

mean

16.0

$17.43 \quad 33.828$ 


\begin{tabular}{|c|c|c|c|c|c|c|c|c|c|c|c|c|}
\hline weather & $\begin{array}{l}\text { air tem- } \\
\text { perature } \\
{ }^{\circ} \mathrm{C}\end{array}$ & $\begin{array}{c}\text { precipi- } \\
\text { tation } \\
\text { mm }\end{array}$ & wind & $\begin{array}{l}\text { water tem- } \\
\text { perature } \\
{ }^{\circ} \mathrm{C}\end{array}$ & $\begin{array}{r}\text { salinity } \\
\% 0\end{array}$ & date & weather & $\begin{array}{l}\text { air tem- } \\
\text { perature } \\
{ }^{\circ} \mathrm{C}\end{array}$ & $\begin{array}{c}\text { precipi- } \\
\text { tation } \\
\mathrm{mm}\end{array}$ & wind & $\begin{array}{l}\text { water tem- } \\
\text { perature } \\
{ }^{\circ} \mathrm{C}\end{array}$ & salinity \\
\hline $\begin{array}{l}o \\
b \\
b \\
o \\
o\end{array}$ & $\begin{array}{l}22.2 \\
19.1 \\
18.4 \\
19.9 \\
21.6\end{array}$ & $\frac{68.3}{\bar{E}}$ & $\begin{array}{r}\mathrm{S} 2 \\
\mathrm{NW} 4 \\
-0 \\
\mathrm{SE} \\
-0\end{array}$ & $\begin{array}{l}21.28 \\
19.99 \\
20.92 \\
19.11 \\
20.10\end{array}$ & $\begin{array}{l}33.822 \\
34.065 \\
33.900 \\
34.437 \\
33.622\end{array}$ & $\begin{array}{l}1 \\
2 \\
3 \\
4 \\
5\end{array}$ & $\begin{array}{r}b \\
b c \\
0 \\
0 \\
b c\end{array}$ & $\begin{array}{l}23.0 \\
22.6 \\
22.4 \\
17.8 \\
20.0\end{array}$ & $\begin{array}{r}\overline{-} \\
\overline{0.1} \\
24.6 \\
19.5\end{array}$ & $\begin{array}{rr}- & 0 \\
\mathrm{NE} & 1 \\
- & 0 \\
\mathrm{NW} & 1 \\
- & 0\end{array}$ & $\begin{array}{l}21.22 \\
21.50 \\
21.28 \\
21.10 \\
21.21\end{array}$ & $\begin{array}{l}34.401 \\
34.321 \\
34.377 \\
33.593 \\
34.085\end{array}$ \\
\hline $\begin{array}{r}\mathrm{b} \\
\mathrm{bc} \\
\mathrm{o} \\
\mathrm{o} \\
\mathrm{o}\end{array}$ & $\begin{array}{l}20.1 \\
21.7 \\
21.4 \\
23.2 \\
19.3\end{array}$ & $\frac{-}{4.0}$ & $\begin{array}{r}\text { WNW } 3 \\
-0 \\
\text { SW } 0 \\
-0\end{array}$ & $\begin{array}{l}19.24 \\
19.86 \\
20.99 \\
21.45 \\
20.80\end{array}$ & $\begin{array}{l}33.579 \\
33.657 \\
33.692 \\
33.947 \\
34.022\end{array}$ & $\begin{array}{r}6 \\
7 \\
8 \\
9 \\
10\end{array}$ & $\begin{array}{l}\mathrm{b} \\
\mathrm{r} \\
\mathrm{o} \\
\mathrm{o} \\
\mathrm{b}\end{array}$ & $\begin{array}{l}22.4 \\
19.8 \\
23.4 \\
22.5 \\
24.0\end{array}$ & $\begin{array}{r}\overline{1.3} \\
58.3 \\
3.2 \\
-\end{array}$ & $\begin{array}{rl}\overline{\mathrm{E}} & 0 \\
\mathrm{SE} & 2 \\
\overline{\mathrm{S}} & 0\end{array}$ & $\begin{array}{l}22.15 \\
21.71 \\
22.18 \\
22.31 \\
22.49\end{array}$ & $\begin{array}{l}33.908 \\
34.004 \\
33.492 \\
33.600 \\
33.444\end{array}$ \\
\hline $\begin{array}{r}\mathbf{o} \\
\mathbf{b c} \\
\mathbf{r} \\
\mathbf{r} \\
\mathrm{d}\end{array}$ & $\begin{array}{l}22.7 \\
23.1 \\
18.2 \\
19.9 \\
21.6\end{array}$ & $\begin{array}{r}\overline{-} \\
13.7 \\
15.5 \\
17.6\end{array}$ & $\begin{array}{l}-0 \\
=0 \\
=0 \\
=0 \\
-0\end{array}$ & $\begin{array}{l}21.42 \\
21.01 \\
21.00 \\
20.50 \\
21.60\end{array}$ & $\begin{array}{l}34.041 \\
34.054 \\
33.386 \\
33.056 \\
33.662\end{array}$ & $\begin{array}{l}11 \\
12 \\
13 \\
14 \\
15\end{array}$ & $\begin{array}{r}o \\
r \\
o \\
b c \\
o\end{array}$ & $\begin{array}{l}27.0 \\
20.8 \\
23.7 \\
23.9 \\
24.2\end{array}$ & $\begin{array}{r}11 \overline{5.5} \\
75.5 \\
\overline{-}\end{array}$ & $\begin{array}{rl}\overline{\text { ENE }} & 0 \\
\mathrm{~S} & 1 \\
- & 0 \\
- & 0\end{array}$ & $\begin{array}{l}22.80 \\
22.12 \\
22.52 \\
22.68 \\
22.70\end{array}$ & $\begin{array}{l}33.893 \\
27.205 \\
32.663 \\
33.477 \\
33.477\end{array}$ \\
\hline $\begin{array}{r}b c \\
b \\
b c \\
c \\
o\end{array}$ & $\begin{array}{l}22.9 \\
18.3 \\
19.1 \\
21.6 \\
21.1\end{array}$ & $\frac{0.7}{\bar{z}}$ & $\begin{array}{rl}\mathrm{SW} & 2 \\
\mathrm{E} & 2 \\
\mathrm{~S} & 0 \\
\mathrm{SSW} & 1\end{array}$ & $\begin{array}{l}22.35 \\
20.90 \\
19.96 \\
21.10 \\
20.49\end{array}$ & $\begin{array}{l}33.531 \\
33.958 \\
32.998 \\
33.874 \\
33.820\end{array}$ & $\begin{array}{l}16 \\
17 \\
18 \\
19 \\
20\end{array}$ & $\begin{array}{r}\mathrm{bc} \\
\mathrm{c} \\
\mathrm{r} \\
\mathrm{r} \\
\mathrm{o}\end{array}$ & $\begin{array}{l}24.5 \\
24.3 \\
22.0 \\
21.5 \\
23.8\end{array}$ & $\begin{array}{r}- \\
60.5 \\
5.7 \\
16.3\end{array}$ & $\begin{array}{r}-0 \\
-0 \\
-0 \\
N W 1 \\
-0\end{array}$ & $\begin{array}{l}22.69 \\
23.39 \\
23.84 \\
22.95 \\
23.15\end{array}$ & $\begin{array}{l}33.423 \\
33.441 \\
31.437 \\
32.809 \\
32.249\end{array}$ \\
\hline $\begin{array}{l}\mathrm{b} \\
\mathrm{b} \\
\mathrm{b} \\
\mathrm{b} \\
\mathrm{bc}\end{array}$ & $\begin{array}{l}20.5 \\
20.7 \\
20.6 \\
22.6 \\
21.6\end{array}$ & $\begin{array}{l}6.1 \\
\text { 二 } \\
-\end{array}$ & $\begin{array}{rr}\text { NW } & 2 \\
\text { NW } & 2 \\
\mathrm{~S} & 1 \\
\mathrm{~S} & 1 \\
- & 0\end{array}$ & $\begin{array}{l}20.65 \\
20.32 \\
20.19 \\
21.59 \\
21.60\end{array}$ & $\begin{array}{l}33.817 \\
34.384 \\
34.375 \\
34.483 \\
34.541\end{array}$ & $\begin{array}{l}21 \\
22 \\
23 \\
24 \\
25\end{array}$ & $\begin{array}{r}\mathrm{c} \\
\mathrm{r} \\
\mathrm{o} \\
\mathrm{bc} \\
\mathrm{b}\end{array}$ & $\begin{array}{l}23.6 \\
21.0 \\
19.7 \\
24.5 \\
23.8\end{array}$ & $\begin{array}{r}\overline{-} \\
25.2 \\
\overline{-}\end{array}$ & $\begin{array}{rl}\mathrm{NW} & 1 \\
\mathrm{NE} & 1 \\
\mathrm{E} & 1 \\
\mathbf{E} & 1 \\
- & 0\end{array}$ & $\begin{array}{l}23.22 \\
22.34 \\
22.00 \\
22.50 \\
23.10\end{array}$ & $\begin{array}{l}32.195 \\
33.098 \\
32.538 \\
32.195 \\
30.877\end{array}$ \\
\hline $\begin{array}{l}26 \\
27 \\
28 \\
29 \\
30 \\
31\end{array}$ & $\begin{array}{l}20.4 \\
22.7 \\
22.4 \\
23.2 \\
21.5 \\
20.5\end{array}$ & $\begin{array}{r}2 \overline{\overline{0}} \\
\overline{\bar{T}} \\
5.0 \\
9.5\end{array}$ & $\begin{array}{r}\overline{S W} 2 \\
\mathrm{SW} 1 \\
\mathrm{NE} 2 \\
\mathrm{NW} 3\end{array}$ & $\begin{array}{l}21.29 \\
21.55 \\
23.84 \\
21.30 \\
21.36 \\
21.55\end{array}$ & $\begin{array}{l}34.032 \\
33.590 \\
34.258 \\
34.406 \\
33.539 \\
34.357\end{array}$ & $\begin{array}{l}26 \\
27 \\
28 \\
29 \\
30\end{array}$ & $\begin{array}{r}\mathrm{bc} \\
\mathrm{r} \\
\mathrm{o} \\
\mathrm{d} \\
\mathrm{b}\end{array}$ & $\begin{array}{l}23.6 \\
19.8 \\
23.9 \\
23.6 \\
26.4\end{array}$ & $\begin{array}{r}\overline{9.2} \\
64.5 \\
0.5 \\
0.9\end{array}$ & 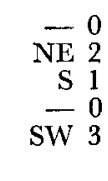 & $\begin{array}{l}22.83 \\
22.17 \\
23.60 \\
23.91 \\
24.70\end{array}$ & $\begin{array}{l}33.025 \\
33.242 \\
33.116 \\
32.809 \\
33.459\end{array}$ \\
\hline ean & 21.0 & & & 20.95 & 33.900 & mean & & 22.8 & & & 22.55 & 32.995 \\
\hline
\end{tabular}


JULY

\begin{tabular}{|c|c|c|c|c|c|c|}
\hline date & weather & $\begin{array}{l}\text { air tem- } \\
\text { perature } \\
{ }^{\circ} \mathrm{C}\end{array}$ & $\begin{array}{c}\text { precipi- } \\
\text { tation } \\
\mathrm{mm}\end{array}$ & wind & $\begin{array}{l}\text { water tem- } \\
\text { perature } \\
{ }^{\circ} \mathrm{C}\end{array}$ & salinity \\
\hline $\begin{array}{l}1 \\
2 \\
3 \\
4 \\
5\end{array}$ & $\begin{array}{l}o \\
b \\
o \\
o \\
o\end{array}$ & $\begin{array}{l}26.5 \\
27.2 \\
27.5 \\
27.2 \\
27.6\end{array}$ & $\begin{array}{l}E \\
\bar{Z} \\
2.5 \\
1.8\end{array}$ & $\begin{array}{rl}\mathrm{S} & 0 \\
\mathrm{NE} & 1 \\
\mathrm{SSW} & 2 \\
\mathrm{SW} & 1\end{array}$ & $\begin{array}{l}24.69 \\
25.29 \\
25.61 \\
25.59 \\
25.74\end{array}$ & $\begin{array}{l}32.971 \\
33.025 \\
32.628 \\
32.935 \\
32.983\end{array}$ \\
\hline $\begin{array}{r}6 \\
7 \\
8 \\
9 \\
10\end{array}$ & $\begin{array}{r}r \\
o \\
b c \\
b c \\
o\end{array}$ & $\begin{array}{l}25.7 \\
26.4 \\
26.6 \\
26.7 \\
26.1\end{array}$ & $\begin{array}{r}20.2 \\
14.6 \\
0.1 \\
-\end{array}$ & $\begin{array}{rr}\mathrm{S} & 1 \\
\mathrm{ESE} & 1 \\
\mathrm{~S} & 2 \\
\mathrm{~S} & 2 \\
\mathrm{SW} & 1\end{array}$ & $\begin{array}{l}26.80 \\
25.41 \\
25.91 \\
25.80 \\
25.35\end{array}$ & $\begin{array}{l}33.062 \\
32.701 \\
31.816 \\
31.581 \\
31.924\end{array}$ \\
\hline $\begin{array}{l}11 \\
12 \\
13 \\
14 \\
15\end{array}$ & $\begin{array}{r}\text { bc } \\
o \\
r \\
b \\
b c\end{array}$ & $\begin{array}{l}26.4 \\
26.9 \\
24.5 \\
26.4 \\
23.6\end{array}$ & $\begin{array}{r}- \\
157.0 \\
72.0 \\
-\end{array}$ & $\begin{array}{rl}\mathrm{S} & 2 \\
\mathrm{SE} & 2 \\
\mathrm{SW} & 2 \\
\mathrm{~S} & 1 \\
\mathrm{NW} & 2\end{array}$ & $\begin{array}{l}26.20 \\
25.82 \\
24.90 \\
24.72 \\
24.55\end{array}$ & $\begin{array}{l}32.069 \\
31.997 \\
29.632 \\
29.181 \\
31.888\end{array}$ \\
\hline $\begin{array}{l}16 \\
17 \\
18 \\
19 \\
20\end{array}$ & $\begin{array}{l}o \\
o \\
b \\
c \\
o\end{array}$ & $\begin{array}{l}22.8 \\
24.9 \\
27.7 \\
28.9 \\
28.3\end{array}$ & $\begin{array}{r}12.0 \\
0.1 \\
2.0 \\
-\end{array}$ & $\begin{array}{rr}-0 \\
- & 0 \\
\mathrm{~S} & 1 \\
\mathrm{ENE} & 2 \\
- & 0\end{array}$ & $\begin{array}{l}23.80 \\
24.75 \\
25.55 \\
26.07 \\
26.00\end{array}$ & $\begin{array}{l}32.953 \\
30.769 \\
33.080 \\
33.296 \\
33.296\end{array}$ \\
\hline $\begin{array}{l}21 \\
22 \\
23 \\
24 \\
25\end{array}$ & $\begin{array}{r}b c \\
b c \\
o \\
b c \\
b\end{array}$ & $\begin{array}{l}29.5 \\
29.9 \\
29.0 \\
27.5 \\
29.1\end{array}$ & $\begin{array}{r}- \\
\overline{0.6} \\
14.6 \\
-\end{array}$ & $\begin{array}{ll}\text { SW } & 2 \\
- & 0 \\
N E & 1 \\
N E & 1 \\
\text { SW } & 1\end{array}$ & $\begin{array}{l}26.12 \\
\\
26.30 \\
26.29 \\
26.62\end{array}$ & $\begin{array}{l}33.080 \\
32.863 \\
33.043 \\
32.610 \\
33.260\end{array}$ \\
\hline $\begin{array}{l}26 \\
27 \\
28 \\
29 \\
30 \\
31\end{array}$ & $\begin{array}{r}b c \\
o \\
b c \\
b \\
b c \\
b c\end{array}$ & $\begin{array}{l}29.8 \\
25.6 \\
25.3 \\
26.2 \\
28.4 \\
27.8\end{array}$ & $\begin{array}{l}\overline{0.5} \\
6.2 \\
- \\
-\end{array}$ & $\begin{array}{r}\text { WE } 1 \\
-0 \\
-0 \\
-0 \\
-0 \\
-0\end{array}$ & $\begin{array}{l}26.95 \\
26.80 \\
27.05 \\
26.91 \\
27.40 \\
27.33\end{array}$ & $\begin{array}{l}33.513 \\
33.766 \\
33.603 \\
32.827 \\
33.513 \\
32.484\end{array}$ \\
\hline mean & & 27.0 & & & 25.88 & 32.527 \\
\hline
\end{tabular}

AUGUST

date weather air tem- precipi- wind water tem- salinity $\underset{{ }^{\circ} \mathrm{C}}{\operatorname{merature}} \stackrel{\mathrm{mm}}{\text { tation }}$

${ }^{\circ} \mathrm{C} \quad \%$

\begin{tabular}{|c|c|c|c|c|}
\hline $\begin{array}{l}27.7 \\
28.2 \\
28.9 \\
28.7 \\
29.2\end{array}$ & $\begin{array}{l}- \\
- \\
-\end{array}$ & $\begin{array}{ll}S & 2 \\
S & 1 \\
- & 0 \\
- & 0 \\
S & 1\end{array}$ & $\begin{array}{l}27.70 \\
27.31 \\
28.09 \\
28.13 \\
29.20\end{array}$ & $\begin{array}{l}32.628 \\
32.502 \\
33.368 \\
33.122 \\
33.359\end{array}$ \\
\hline $\begin{array}{l}28.9 \\
28.6 \\
29.1 \\
29.0 \\
27.5\end{array}$ & $\bar{E} \frac{}{38.0}$ & $\begin{array}{rr}- & 0 \\
\mathrm{NNE} & 1 \\
- & 0 \\
\mathrm{SW} & 1 \\
\mathrm{~S} & 2\end{array}$ & $\begin{array}{l}28.82 \\
28.38 \\
28.82 \\
28.97 \\
28.01\end{array}$ & $\begin{array}{l}33.369 \\
33.398 \\
33.276 \\
33.492 \\
32.779\end{array}$ \\
\hline $\begin{array}{l}23.8 \\
24.6 \\
27.3 \\
27.9 \\
27.8\end{array}$ & $\begin{array}{r}3.7 \\
61.0 \\
14.7 \\
1.9 \\
\end{array}$ & 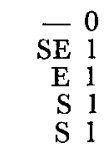 & $\begin{array}{l}27.35 \\
27.72 \\
27.80 \\
27.72 \\
27.61\end{array}$ & $\begin{array}{l}31.778 \\
30.325 \\
31.854 \\
32,346 \\
32.294\end{array}$ \\
\hline $\begin{array}{l}28.2 \\
28.7 \\
28.4 \\
28.3 \\
29.1\end{array}$ & $\frac{E}{5.5}$ & $\begin{array}{rr}S & 1 \\
S & 1 \\
S W & 2\end{array}$ & $\begin{array}{l}27.60 \\
27.37 \\
27.83 \\
28.07 \\
28.42\end{array}$ & $\begin{array}{l}33.195 \\
33.694 \\
33.696 \\
33.772 \\
32.358\end{array}$ \\
\hline $\begin{array}{l}27.8 \\
23.6 \\
22.9 \\
25.6 \\
24.0\end{array}$ & $\begin{array}{r}2.0 \\
61.0 \\
27.2 \\
11.0\end{array}$ & $\begin{array}{l}\mathrm{SE} 2 \\
-0 \\
-0 \\
-0 \\
-0\end{array}$ & $\begin{array}{l}28.01 \\
27.10 \\
26.30 \\
26.02 \\
26.60\end{array}$ & $\begin{array}{l}32.521 \\
31.437 \\
29.693 \\
32.250 \\
33.050\end{array}$ \\
\hline $\begin{array}{l}26.8 \\
26.6 \\
27.8 \\
27.8 \\
27.5 \\
29.0\end{array}$ & $\begin{array}{r}21.8 \\
6.4 \\
- \\
- \\
-\end{array}$ & $\begin{array}{rr}\mathrm{S} & 2 \\
\mathrm{NW} & 2 \\
- & 0 \\
\mathrm{SW} & 1 \\
\mathrm{~S} & 1 \\
\mathrm{~S} & 1\end{array}$ & $\begin{array}{l}26.70 \\
26.80 \\
27.30 \\
27.32 \\
27.56 \\
28.10\end{array}$ & $\begin{array}{l}32.656 \\
33.417 \\
33.136 \\
33.163 \\
32.722 \\
32.895\end{array}$ \\
\hline 27.4 & & & 27.70 & 32.695 \\
\hline
\end{tabular}


SEPTEMBER

\begin{tabular}{|c|c|c|c|c|c|c|}
\hline date & weather & $\begin{array}{l}\text { air tem- } \\
\text { perature } \\
{ }^{\circ} \mathrm{C}\end{array}$ & $\begin{array}{l}\text { precipi- } \\
\text { tation } \\
\qquad \mathrm{mm}\end{array}$ & wind & $\begin{array}{l}\text { water tem- } \\
\text { perature } \\
{ }^{\circ} \mathrm{C}\end{array}$ & $\begin{array}{r}\text { salinity } \\
\text { \%o }\end{array}$ \\
\hline $\begin{array}{l}1 \\
2 \\
3 \\
4 \\
5\end{array}$ & $\begin{array}{r}b c \\
b c \\
b \\
b c \\
b\end{array}$ & $\begin{array}{l}29.1 \\
27.3 \\
27.2 \\
28.4 \\
27.9\end{array}$ & $\begin{array}{l}E \\
E\end{array}$ & $\begin{array}{rr}\text { NW } & 1 \\
- & 0 \\
- & 0 \\
\text { NNE } & 2 \\
\text { S } & 1\end{array}$ & $\begin{array}{l}28.65 \\
27.20 \\
27.30 \\
27.50 \\
27.39\end{array}$ & $\begin{array}{l}33.099 \\
33.449 \\
33.510 \\
33.236 \\
33.227\end{array}$ \\
\hline $\begin{array}{r}6 \\
7 \\
8 \\
9 \\
10\end{array}$ & $\begin{array}{r}\mathrm{bc} \\
\mathrm{o} \\
\mathrm{bc} \\
0 \\
\mathrm{~b}\end{array}$ & $\begin{array}{l}31.0 \\
24.5 \\
29.4 \\
26.0 \\
25.1\end{array}$ & $\begin{array}{r}85.0 \\
3.1 \\
50.5 \\
-\end{array}$ & $\begin{array}{rr} & 0 \\
\mathrm{NE} & 1 \\
\mathrm{~S} & 1 \\
\mathrm{SE} & 2 \\
\mathrm{NW} & 1\end{array}$ & $\begin{array}{l}27.75 \\
27.01 \\
27.22 \\
26.77 \\
26.92\end{array}$ & $\begin{array}{l}33.336 \\
32.792 \\
33.093 \\
32.617 \\
32.240\end{array}$ \\
\hline $\begin{array}{l}11 \\
12 \\
13 \\
14 \\
15\end{array}$ & $\begin{array}{r}\mathrm{b} \\
\mathrm{bc} \\
\mathrm{bc} \\
\mathrm{p} \\
\mathrm{r}\end{array}$ & $\begin{array}{l}26.0 \\
25.7 \\
24.9 \\
24.6 \\
22.5\end{array}$ & $\begin{array}{r}70.3 \\
\overline{1.7} \\
40.5\end{array}$ & $\begin{array}{rl}- & 0 \\
\overline{N E} & 0 \\
\mathrm{NE} & 2 \\
- & 0\end{array}$ & $\begin{array}{l}26.31 \\
26.56 \\
25.90 \\
25.70 \\
25.30\end{array}$ & $\begin{array}{l}32.867 \\
32.829 \\
32.617 \\
32.932 \\
31.159\end{array}$ \\
\hline $\begin{array}{l}16 \\
17 \\
18 \\
19 \\
20\end{array}$ & $\begin{array}{r}r \\
b c \\
b \\
b c \\
b c\end{array}$ & $\begin{array}{l}22.4 \\
25.9 \\
25.1 \\
23.1 \\
23.2\end{array}$ & $\begin{array}{r}14.0 \\
97.0 \\
0.2 \\
7.3 \\
\end{array}$ & $\begin{array}{ll}N W & 2 \\
N W & 2 \\
N W & 1 \\
-1 & 0 \\
N W & 1\end{array}$ & $\begin{array}{l}25.41 \\
25.41\end{array}$ & $\begin{array}{l}32.949 \\
32.535 \\
32.202\end{array}$ \\
\hline $\begin{array}{l}21 \\
22 \\
23 \\
24 \\
25\end{array}$ & $\begin{array}{r}\mathrm{bc} \\
\mathrm{b} \\
\mathrm{bc} \\
\mathrm{o} \\
\mathbf{0}\end{array}$ & $\begin{array}{l}24.1 \\
25.9 \\
26.2 \\
24.6 \\
21.6\end{array}$ & E & $\begin{array}{ll}- & 0 \\
\bar{E} & 0 \\
\mathrm{E} & 1 \\
\overline{\mathrm{N}} & 0\end{array}$ & $\begin{array}{l}25.50 \\
25.09 \\
26.00 \\
25.43 \\
24.46\end{array}$ & $\begin{array}{l}32.940 \\
32.399 \\
33.122 \\
32.919 \\
32.622\end{array}$ \\
\hline $\begin{array}{l}26 \\
27 \\
28 \\
29 \\
30\end{array}$ & $\begin{array}{r}\mathrm{b} \\
\mathrm{b} \\
\mathrm{o} \\
\mathrm{bc} \\
\mathrm{bc}\end{array}$ & $\begin{array}{l}21.2 \\
21.6 \\
22.9 \\
23.9 \\
21.5\end{array}$ & $\bar{E}$ & $\begin{aligned} &-0 \\
&-0 \\
&-0 \\
& \text { NW } 2 \\
& \text { NW } 2\end{aligned}$ & $\begin{array}{l}24.10 \\
23.30 \\
23.59 \\
23.80 \\
23.31\end{array}$ & $\begin{array}{l}32.828 \\
32.294 \\
32.792 \\
32.245 \\
32.874\end{array}$ \\
\hline mean & & 25.1 & & & 25.92 & 32.776 \\
\hline
\end{tabular}

OGTOBER

\begin{tabular}{|c|c|c|c|c|c|c|}
\hline date & weather & $\begin{array}{l}\text { air tem- } \\
\text { perature } \\
{ }^{\circ} \mathrm{C}\end{array}$ & $\begin{array}{c}\text { precipi- } \\
\text { tation } \\
\mathrm{mm}\end{array}$ & wind & $\begin{array}{l}\text { water tem- } \\
\text { perature } \\
{ }^{\circ} \mathrm{C}\end{array}$ & $\begin{array}{r}\text { salinity } \\
\% 0\end{array}$ \\
\hline 1 & bc & 20.6 & - & -0 & 23.48 & 33.094 \\
\hline 2 & b & 22.4 & - & NW 2 & 23.12 & 32.657 \\
\hline 3 & b & 23.3 & - & $\mathrm{NE} 1$ & 23.32 & 33.003 \\
\hline 4 & c & 25.8 & - & SW 1 & 23.90 & 32.460 \\
\hline 5 & $\mathrm{bc}$ & 20.7 & 5.5 & NW 2 & 22.67 & 32.062 \\
\hline 6 & $\mathrm{bc}$ & 22.6 & - & NW 3 & 22.25 & 33.041 \\
\hline 7 & $\mathrm{~b}$ & 21.2 & - & -0 & 22.95 & 33.850 \\
\hline 8 & b & 21.6 & - & -0 & 22.88 & 33.867 \\
\hline 9 & $\mathrm{c}$ & 23.3 & - & -0 & 23.65 & 33.883 \\
\hline 10 & $b c$ & 21.9 & - & -0 & 22.66 & 32.333 \\
\hline 11 & $\mathrm{bc}$ & 21.9 & 9.5 & NW 1 & 23.22 & 32.735 \\
\hline 12 & b & 21.8 & - & NNW 3 & 22.35 & 33.103 \\
\hline 13 & $\tilde{b}$ & 19.9 & - & NW 1 & 21.63 & 33.342 \\
\hline 14 & b & 20,3 & 一 & NW 1 & 21.46 & 33.062 \\
\hline 15 & b & 21.2 & - & -0 & 21.76 & 33.140 \\
\hline 16 & b & 21.8 & 11.8 & NW 2 & 22.45 & 33.214 \\
\hline 17 & $c$ & 16.6 & - & -0 & 21.72 & 33.395 \\
\hline 18 & c & 19.6 & - & -0 & 21.88 & 33.619 \\
\hline 19 & $r$ & 19.1 & 3.8 & NE 2 & 22.24 & 33.330 \\
\hline 20 & o & 21.6 & 13.5 & NE 2 & 22.64 & 33.459 \\
\hline 21 & $\mathrm{bc}$ & 23.0 & - & -0 & 22.64 & 33.823 \\
\hline 22 & $o$ & 19.9 & 19.1 & NW 2 & 22.16 & 33.540 \\
\hline 23 & $\mathrm{bc}$ & 18.5 & - & NW 2 & 22.60 & 33.940 \\
\hline 24 & b & 17.5 & - & -0 & 20.64 & 33.916 \\
\hline 25 & b & 19.1 & - & -0 & 21.04 & 33.919 \\
\hline 26 & o & 19.1 & - & -0 & 21.07 & 33.792 \\
\hline 27 & o & 19.6 & 20.2 & NW 1 & 21.62 & 33.473 \\
\hline 28 & b & 16.4 & - & -0 & 20.77 & 33.572 \\
\hline 29 & $\mathrm{bc}$ & 18.6 & -- & -0 & 21.22 & 33.636 \\
\hline 30 & $\mathrm{bc}$ & 18.1 & - & -0 & 21.27 & 33.827 \\
\hline 31 & bc & 17.1 & 9.2 & NW 2 & 20.71 & 33.782 \\
\hline mean & & 20.5 & & & 22.13 & 33.351 \\
\hline
\end{tabular}


NOVEMBER

date weather air tem- precipi- wind perature tem- salinity ${ }^{\circ} \mathrm{C} \quad \mathrm{mm}$

$$
\mathrm{mm}
$$$$
\text { perature }{ }^{\circ} \mathrm{C}
$$$$
\begin{aligned}
& 1 \\
& 2 \\
& 3 \\
& 4 \\
& 5 \\
& 6
\end{aligned}
$$$$
\begin{array}{r}
6 \\
7 \\
8 \\
9 \\
10
\end{array}
$$

N 1

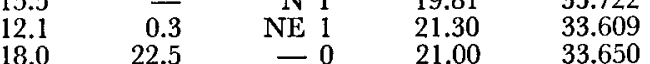

20.14

19.81

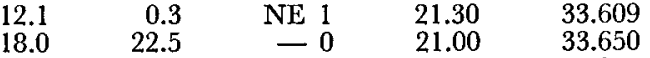

33.809

33.722
33.609

$\begin{array}{lrrrr}18.0 & 22.5 & -{ }_{0}^{0} & 21.00 & 33.650 \\ 19.6 & - & N W 1 & 20.75 & 33.915\end{array}$

20.7

20.7
18.3
14.3
14.0
16.8

$\overline{1.0}$
3.2

$\begin{array}{rrr}\text { S } 1 & 20.95 \\ \text { NW } & 2 & 20.13\end{array}$

$\begin{array}{rrr}\text { NW } 2 & 20.13 & 33.784 \\ \text { N } 1 & 19.60 & 33.769\end{array}$

33.909

33.909
33.784

N $19.60 \quad 33.787$

$20.10 \quad 33.964$

$16.5 \quad 18.1$

NW

19.07

$19.18 \quad 33.428$

33.402
33.428
33.392

33.392

$\begin{array}{rll}\text { NW } 1 & 19.78 & 33.762 \\ \text { E } 1 & 19.91 & 32.720\end{array}$

$18.2 \quad 48.2$

(1)

$20.61 \quad 34.436$

$\begin{array}{rrr}\text { NW } 3 & 17.82 & 33.417 \\ -0 & 16.97 & 32.750\end{array}$

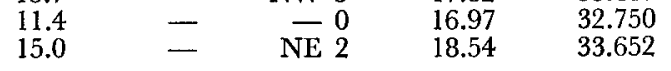

bc 12.1

12.1
10.3
10.2
10.1

-

WNW 4

18.40

34.044

34.044
34.055

$\begin{array}{rrr}\text { N 3 } & 16.79 & 34.055 \\ \text { NW 1 } & 17.26 & 33.912 \\ \text { NWW 3 } & 17.74 & 33.938\end{array}$

bc

$\begin{array}{lll}10.7 & - & \text { NW } 2 \\ 14.4 & 0.2 & \text { SW } 2\end{array}$

$\begin{array}{ll}17.74 & 33.938 \\ 16.12 & 34.864\end{array}$

$16.73 \quad 34.534$

$16.60 \quad 34.391$

$15.61 \quad 34.294$

9.6
13.8
12.1

$\underline{\underline{E}}$

NW 1

NE 1
-0

$\begin{array}{ll}15.07 & 34.292 \\ 15.76 & 33.743\end{array}$

18.64

33.826

\section{DEGEMBER}

date weather air tem- $\begin{aligned} & \text { precipi- wind } \\ & \text { perature } \\ & \text { tation }\end{aligned}$ perature $\quad \begin{gathered}\text { tation } \\ { }^{\circ} \mathrm{G}\end{gathered}$

$$
\text { mm }
$$

$\begin{array}{ll}\text { WNW } 4 & 14.26\end{array}$

$$
\begin{array}{lllll}
6.8 & - & \text { WNW } 4 & 14.26 & 34.093 \\
9.3 & - & \text { WNW } 4 & 12.99 & 34.140 \\
8.6 & - & \text { NE } 1 & 15.10 & 34.016
\end{array}
$$

$\begin{array}{llll}8.6 & - & -15.10 & 34.016\end{array}$

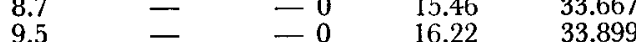

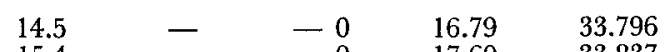

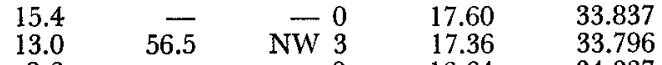

$\begin{array}{rrrr}8.6 & - & -0 & 16.64\end{array}$

$\begin{array}{lllll}10.8 & - & -0 & 18.12 & 34.359\end{array}$

$\begin{array}{llrrr}10.0 & - & -0 & 18.59 & 34.350\end{array}$

$\begin{array}{rrrrr}17.5 & 4.1 & \text { SSE } 4 & 17.64 & 34.193 \\ 6.5 & 26.8 & \text { WNW } 4 & 14.65 & 34.349\end{array}$

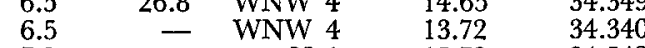

$\begin{array}{rrrrr}7.9 & - & \text { WN } & 15.79 & 34.542\end{array}$

$\begin{array}{lllll}7.8 & - & \text { NW } 1 & 16.87 & 34.432\end{array}$

$\begin{array}{lllll}14.2 & 1.2 & \text { NW } 2 & 17.62 & 34.226\end{array}$

$\begin{array}{llll}7.9 & - & \text { NW } 2 & 14.94\end{array}$

$\begin{array}{llll}8.6 & \text { NE } 2 & 14.74 & 34.222\end{array}$

$\begin{array}{rrrrr}7.6 & - & \text { NE } 1 & 15.80 & 34.489 \\ 12.3 & 1.8 & \text { NE } 1 & 16.85 & 34.370\end{array}$

$\begin{array}{lllll}12.3 & 1.8 & \text { NE } 1 & 16.85 & 34.370 \\ 14.4 & 33.9 & \text { N } 1 & 18.82 & 34.352\end{array}$

$\begin{array}{lllll}11.3 & - & \text { NW } 3 & 16.84 & 34.522\end{array}$

$10.3 \quad-\quad N W 1 \quad 16.95 \quad 34.521$

$\begin{array}{rrrrr}9.1 & - & -0 & 16.56 & 34.513 \\ 13.8 & - & \mathrm{NE} 1 & 17.32 & 34.570\end{array}$

$\begin{array}{lllll}13.8 & -\overline{N E} & 1 & 17.32 & 34.570 \\ 10.8 & 10.1 & -0 & 17.47 & 34.522\end{array}$

$\begin{array}{lllll}6.9 & 1.9 & \text { NW } 3 & 14.90 & 34.523 \\ 6.9 & & \text { NW } 1 & 16.60 & 34.578\end{array}$

10.0

$16.30 \quad 34.284$ 